\title{
AS ESPÉCIES DE ISOPODA (CRUSTACEA: CYMOTHOIDEA) PARASITAS DE SERRASALMUS ALTISPINIS MERCKX, JÉGU \& SANTOS, 2000 (CHARACIFORMES: SERRASALMIDAE) COLETADAS EM LAGOS DE VÁRZEA DA AMAZÔNIA, BRASIL
}

\author{
Germán A. M. MOREY ${ }^{1}$, Hellen P. SANTANA ${ }^{1}$, José C. O. MALTA ${ }^{1}$ \\ 1 Instituto Nacional de Pesquisas da Amazônia, Laboratório de Parasitologia de Peixes Av. André Araújo 2936, Petrópolis, \\ CEP-69.067-375, Manaus, Amazonas, Brasil. E-mail: germantiss1106@gmail.com
}

\section{RESUMO}

As espécies de Isopoda da família Cymothoidae são encontradas parasitando a superfície do corpo, nadadeiras, cavidade bucal e branquial dos peixes, alimentando-se do sangue, muco, epitélio e tecido subcutâneo. Na Amazônia brasileira existem poucos estudos referentes à distribuição destes parasitas e seus hospedeiros. Assim, o objetivo do presente trabalho foi conhecer as espécies de Isopoda que ocorrem em $S$. altispinis coletadas em lagos de várzea da Amazônia brasileira. Foi examinada a superfície externa do corpo, nadadeiras, cavidade bucal e branquial, parede interna do opérculo e aberturas no abdômen de $60 \mathrm{~S}$. altispinis. Os indivíduos encontrados foram coletados com pincéis finos, estiletes, pinças e foram fixados e conservados em etanol $70^{\circ} \mathrm{GL}$ glicerinado a $10 \%$. Foram identificadas duas espécies parasitando as brânquias: 16 indivíduos de Anphira branchialis, Thatcher, 1993 com uma prevalência de 13.33\% e um indivíduo de Vanamea symetrica (Van Name, 1925) com uma prevalência de $1.67 \%$. Os dados obtidos no presente trabalho aumentam o conhecimento das espécies parasitas de $S$. altispinis, sendo reportadas duas novas ocorrências de A. branquialis e $V$. symetrica em um novo hospedeiro.

PALAVRAS CHAVE: Cymothoidae, Isopoda, Lagos de várzea, Parasitas, Serrasalmus altispinis.

\section{THE SPECIES OF ISOPODA (CRUSTACEA: CYMOTHOIDEA) PARASITES OF Serrasalmus altispinis MERCKX, JÉGU E SANTOS, 2000 (CHARACIFORMES: SERRASALMIDAE) COLLECTED IN AMAZON FLOODPLAIN LAKES, BRAZIL}

\begin{abstract}
The Isopoda species belonging to the Cymothoidae family are found parasitizing the body surface, fins, oral cavity and gill of fish, feeding on blood, mucus, epithelial and subcutaneous tissue. In the Brazilian Amazon there are few studies on the distribution of these parasites and their hosts. Thus, the objective of this study was to identify the Isopoda species occurring in S. altispinis collected in Brazilian Amazon floodplain lakes. It was examined the outer surface of the body, fins, buccal and branchial cavity, inner wall of the operculum and openings in the abdomen of $60 \mathrm{~S}$. altispinis. The individuals found were collected with fine brushes, stilletto, tweezers and fixed and preserved in ethanol $70{ }^{\circ} \mathrm{GL}$ with $10 \%$ glycerin. There were identified two species parasitizing the gills: 16 individuals of Anphira branchialis, Thatcher, 1993, with a prevalence of $13.33 \%$ and an individual of Vanamea symetrica (Van Name, 1925) with a prevalence of $1.67 \%$. The data obtained in this study increase the knowledge of the parasitic species in S. altispinis, being reported two new occurrences of $A$. branquialis and $V$. symetrica in a new host.
\end{abstract}

KEYWORDS: Cymothoidae, floodplain lakes, Isopoda, Parasites, Serrasalmus altispinis. 


\section{INTRODUÇÃO}

Os crustáceos que parasitam os peixes são considerados ectoparasitos, mesmo quando sua localização não é perceptível no exterior do indivíduo (Eiras et al., 2000). Os crustáceos parasitas chamam a atenção por serem quase sempre visíveis e apresentarem uma ampla variação no tamanho e na forma. Além disso, sua ação sobre os peixes pode ser direta, ou indireta, podendo funcionar como vetores de doenças causadas por bactérias e fungos (Möller \& Anders, 1986).

Os principais grupos de crustáceos parasitos são os copépodos, branquiúrus e isópodos. A ordem Isopoda pertence à Classe Malacostraca e inclui pelo menos cinco famílias de parasitos de peixes. $\mathrm{Na}$ subordem Flabellifera, encontra-se a família Cymothoidae, cujas espécies são adaptadas ao parasitismo (Eiras et al., 2000).

Usando sete pares de pernas em forma de gancho e peças bucais especializadas, estes parasitas se aderem na superfície do corpo, nadadeiras, cavidade bucal e branquial dos peixes (Brusca et al., 2001). Os machos são sempre menores que as fêmeas (Thatcher, 1993). A cópula e a inseminação ocorrem dentro do hospedeiro (Brusca, 1978). São hematófagos, alimentando-se principalmente do sangue do hospedeiro, sendo capazes de se alimentar também do muco, epitélio e tecido subcutâneo (Ramdane et al., 2007).

Na América do Sul 187 espécies de Isopoda são conhecidas como parasitas de peixes marinhos e de água doce (Luque \& Poulin, 2007). A bacia Amazônica, uma das maiores do mundo, ocupa 40.2 $\%$ da América do Sul, refletindo uma alta diversidade de hospedeiros e parasitas (Luque \& Poulin, 2007). No Brasil, Luque et al. (2013) proporcionaram uma lista de espécies de Isopoda que incluem 23 espécies parasitas de peixes de água doce. Desde então, diversas espécies tem sido registradas, proporcionando mais evidencias da riqueza de isópodes no Brasil, que apresenta mais de 4.000 peixes de água doce distribuídas em mais de 50 famílias (Eiras et al., 2010; Froese \& Pauly, 2014).

Serrasalmus altispinis Merckx, Jégu \& Santos, 2000 foi descrita para o Rio Pitinga na Bacia do Rio Uatumã no Estado do Amazonas e ocorre na Bacia do Rio Amazonas. Pode alcançar até $19 \mathrm{~cm}$ de comprimento padrão e habita lagos e rios de água branca, sendo capturada juntamente com $S$. rhombeus próximo à vegetação aquática e na floresta alagada (Claro-Jr., 2003).

Para a fauna parasitaria de $S$. altispinis só foi reportado um trabalho referente às espécies de copépodes que a parasitam (Morey \& Malta, 2016). Devido às escassas informações sobre a fauna parasitaria deste peixe, o objetivo do presente trabalho foi conhecer as espécies de Isopoda que ocorrem em $S$. altispinis coletadas em lagos de várzea na Amazônia brasileira e, contribuindo com maior conhecimento dos isópodes parasitas deste hospedeiro, que são pela primeira vez abordada neste trabalho

\section{MATERIAIS E MÉTODOS}

Nos meses de março, junho, setembro e dezembro de 2013 foram capturados 60 S. altispinis em cinco lagos de várzea do Rio Solimões. Lago Baixio $\left(03^{\circ} 17^{\prime} 27.2^{\prime \prime} \mathrm{S} / 60^{\circ} 04^{\prime} 29.6^{\prime \prime} \mathrm{O}\right)$ no município de Iranduba, Preto $\left(03^{\circ} 21^{\prime} 17.1^{\prime \prime S} / 60^{\circ} 37^{\prime} 28.6^{\prime \prime} \mathrm{O}\right)$ no município de Manacapuru. Lago Ananá (035' 54.8"S/ 61 $\left.{ }^{\circ} 40^{\prime} 18.4^{\prime \prime O}\right)$ em Anori. Lago Araçá ( $\left.\mathrm{S} 03^{\circ} 45^{\prime} 04.3^{\prime \prime} \mathrm{S} / 62^{\circ} 21^{\prime} 25.9^{\prime \prime} \mathrm{O}\right)$ em Codajás. Lago Maracá (0350'32.8"S/ 62³4'32.4"O) em Coari. E um lago no Rio Purus, o Lago São Tomé $\left(03^{\circ} 49^{\prime}\right.$ 39.0"S/ 61² $25^{\prime} 24.6^{\prime \prime} \mathrm{O}$ ).

Os peixes foram capturados em redes de espera de $20 \mathrm{~m}$ de comprimento por $2 \mathrm{~m}$ de altura com malhas variando de 30 a $100 \mathrm{~mm}$ entre nós adjacentes.

A coleta dos parasitas foi feita examinando a superfície externa do corpo, nadadeiras, cavidade bucal e branquial, parede interna do opérculo e aberturas no abdômen dos hospedeiros.

Os indivíduos encontrados foram coletados com pincéis finos, estiletes e pinças. Fixados e conservados em etanol $70^{\circ}$ GL glicerinado a $10 \%$ (Amato et al., 1991) para posterior identificação no Laboratório de Parasitologia de Peixes (LPP) do Instituto de Pesquisas da Amazônia (INPA).

Para o estudo de Isopoda os apêndices foram dissecados com estiletes, clarificados com fenol puro e foram feitas lâminas provisórias. Para identificar as espécies foram utilizadas as características morfológicas das descrições originais das espécies (Thatcher, 1993). Amostras testemunhas foram depositadas na coleção do Instituto Nacional de Pesquisas da Amazônia (INPA), Manaus - Brasil.

Os índices parasitários calculados foram: Prevalência $(\mathrm{P} \%=\mathrm{npp} / \mathrm{n} \times 100)$, onde $\mathrm{npp}=$ número de peixes parasitados, e $\mathrm{n}=$ número de peixes examinados; Intensidade $(\mathrm{I}=$ número mínimo e máximo de parasitas registrados); Intensidade média $(\operatorname{Im}=$ tpp / npp $)$, onde tpp = número total de parasitas na amostra e npp = número de peixes parasitados; Abundância média $(\mathrm{Am}=\mathrm{tpp} / \mathrm{ntp})$, onde tpp = número total de parasitas na amostra e ntp 
$=$ número total de peixes parasitados ou não na amostra (Bush et al., 1997).

Para determinar uma possível influência negativa no desenvolvimento dos peixes parasitados por isópodes, foi calculado o fator de condição (Kn). Os logaritmos dos valores de comprimento padrão (Ls) e de peso total (Wt) de cada indivíduo hospedeiro foram ajustados à curva da relação $\mathrm{Wt} / \mathrm{Ls}$ e foram estimados os valores dos coeficientes de regressão $a$ e $b$.

Os valores de $a$ e $b$ foram utilizados nas estimativas dos valores teoricamente esperados de peso corporal (We) pela utilização da equação: $\mathrm{We}=\mathrm{a} * \mathrm{Ltb}$. Foi calculado, então, o fator de condição relativo $(\mathrm{Kn})$ que corresponde ao quociente entre o peso observado e o peso teoricamente esperado para um determinado comprimento $(\mathrm{Kn}=\mathrm{Wt} / \mathrm{We})$ (Le Cren, 1951).

\section{RESULTADOS}

Foram analisados $60 \mathrm{~S}$. altispinis com comprimento padrão de $13.34 \mathrm{~cm} \pm 3.62$ e peso $62.87 \mathrm{~g} \pm 32.20$. Dos parasitas registrados, duas espécies foram identificadas: 16 indivíduos de Anphira branchialis, Thatcher (1993), sendo nove machos e sete fêmeas e um macho de Vanamea symetrica (Van Name, 1925). Todos os indivíduos foram encontrados parasitando as brânquias dos hospedeiros (Figura 1). Os índices parasitários são apresentados na tabela 1.

No lago Baixio foram registrados seis indivíduos de A. branchialis; no Ananá foram registrados também seis indivíduos de $A$. branchialis, no lago Araçá quatro $A$. branchialis e um exemplar de $V$. symetrica. $\mathrm{O}$ fator de condição relativo $(\mathrm{Kn})$ dos peixes parasitados variou de 0.39 a 1.77 , com média de $0.99 \pm 0.47$.

Tabela 1. Índices parasitários das espécies de isópodes parasitas de $S$. altispinis coletadas em lagos de várzea da Amazônia, Brasil. $\mathrm{PE}=$ peixes examinados, $\mathrm{PP}=$ peixes parasitados, $\mathrm{P}(\%)=$ prevalência, $\mathrm{I}=$ intensidade de infestação, $\mathrm{IM}$ = intensidade média de infestação, $\mathrm{AM}=$ abundância média de infestação.

\begin{tabular}{ccccccc}
\hline Espécie & PE & PP & P(\%) & I & Im & Am \\
\hline Anphira branchialis & 60 & 8 & 13.33 & $16(2)$ & 2 & 0.27 \\
Vanamea symetrica & 60 & 1 & 1.67 & 1 & 1 & 0.02 \\
\hline
\end{tabular}
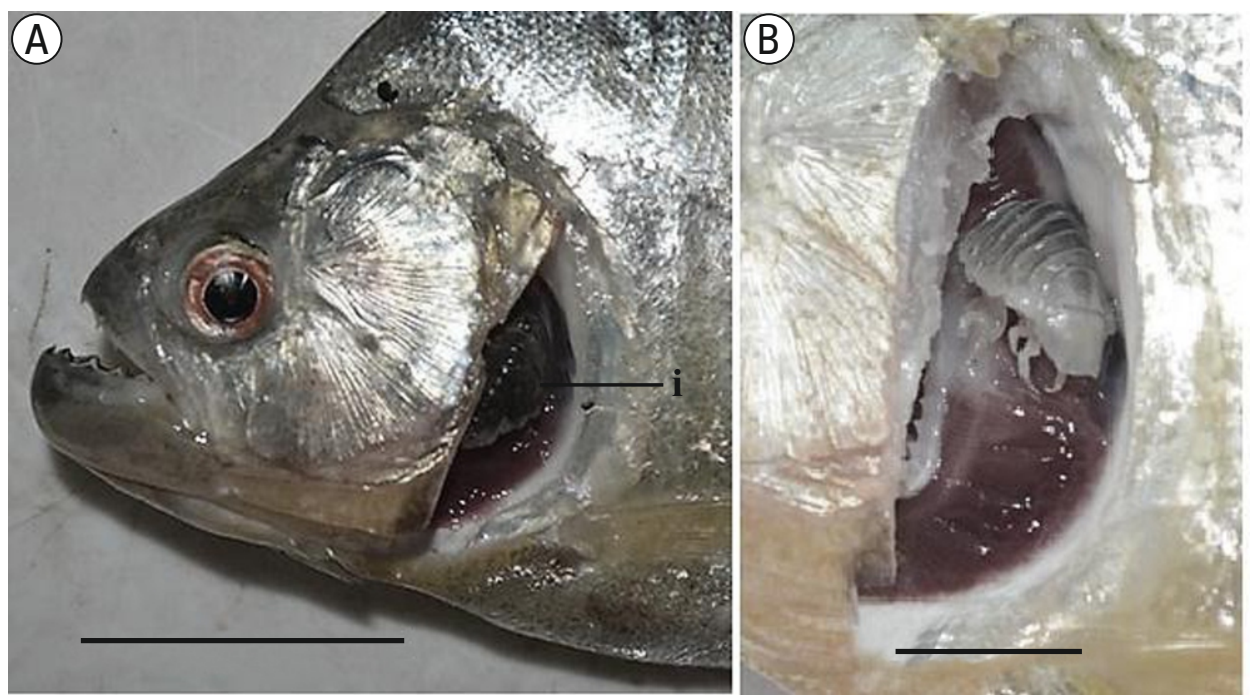

Figura 1. S. altispinis parasitado por $A$. branquialis. A e B fêmea parasitando a cavidade branquial. Escala da barra $A=3 \mathrm{~cm}, B=1 \mathrm{~cm}$. 


\section{DESCRIÇÃO DAS ESPÉCIES REGISTRADAS:}

Anphira branchialis (Figura 2).

Fêmea: Descrição baseada em sete indivíduos coletados e medidos em $\mu \mathrm{m}$ : comprimento do corpo variando entre 11.47-15.61 (13,60) e largura de 7.098.91 (7.79). Corpo mais comprido que largo, pereonito 4 o mais largo. Dorso do pereon altamente convexo, cor variando do quase branco ao marrom escuro, melanóforos pequenos. Cefalon imerso no pereonito 1 , com rotação ventral, margem frontal arredondada, olhos proeminentes. Antênulas e antenas subiguais em comprimento, antênulas suavemente comprimidas, com 8 segmentos; antenas subcilíndricas com 9 segmentos. Labro projetando ventralmente sobre a mandíbula; mandíbula romba, arredondada em forma de pé, sem incisivo, lábio com lobo anexados a mandíbula. Maxílula com 3 espinhos recurvados terminais e 2 subterminais. Maxila bilobada com um espinho recurvado em cada lobo. Palpo do maxilípede com 2 espinhos recurvados 1 terminal e 1 subterminal.
Pereonitos 1 cerca de duas vezes o comprimento do 2-6, 7 metade do comprimento do 6. Coxa 7 estendendo para o pleotelson cobrindo todos os pleonitos lateralmente. Pereiópodos curtos, 2-6 subiguais em comprimento, sendo o número $1 \mathrm{o}$ menor e o número 7 o maior, todos terminados em uma garra curva semelhante a um dáctilo. Urópodo delgado, ramos pontudos, subiguais, não atingindo a margem posterior do pleotelson. Pleópodos lamelares com extremidades arredondadas. Pleotelson sem quilha, arredondados posteriormente. Pleotelson bastante arredondado posteriormente, medindo de 2.84-3.50 $(3,26)$ de comprimento por 4.27-5.03 (4.67) de largura.

Macho: Descrição baseada em nove indivíduos coletados e medidos em mm. Similares à fêmea mais de menor tamanho e menos convexo dorsalmente. Urópodos mais curtos e mais arredondados. Comprimento do corpo variando entre 7.52-10.43 (9.08) e largura de 3.89- 5.41 (4.63). Pleotelson medindo de 1.44-2,70 (2.13) de comprimento por 2.85-3.40 (3.18) de largura.
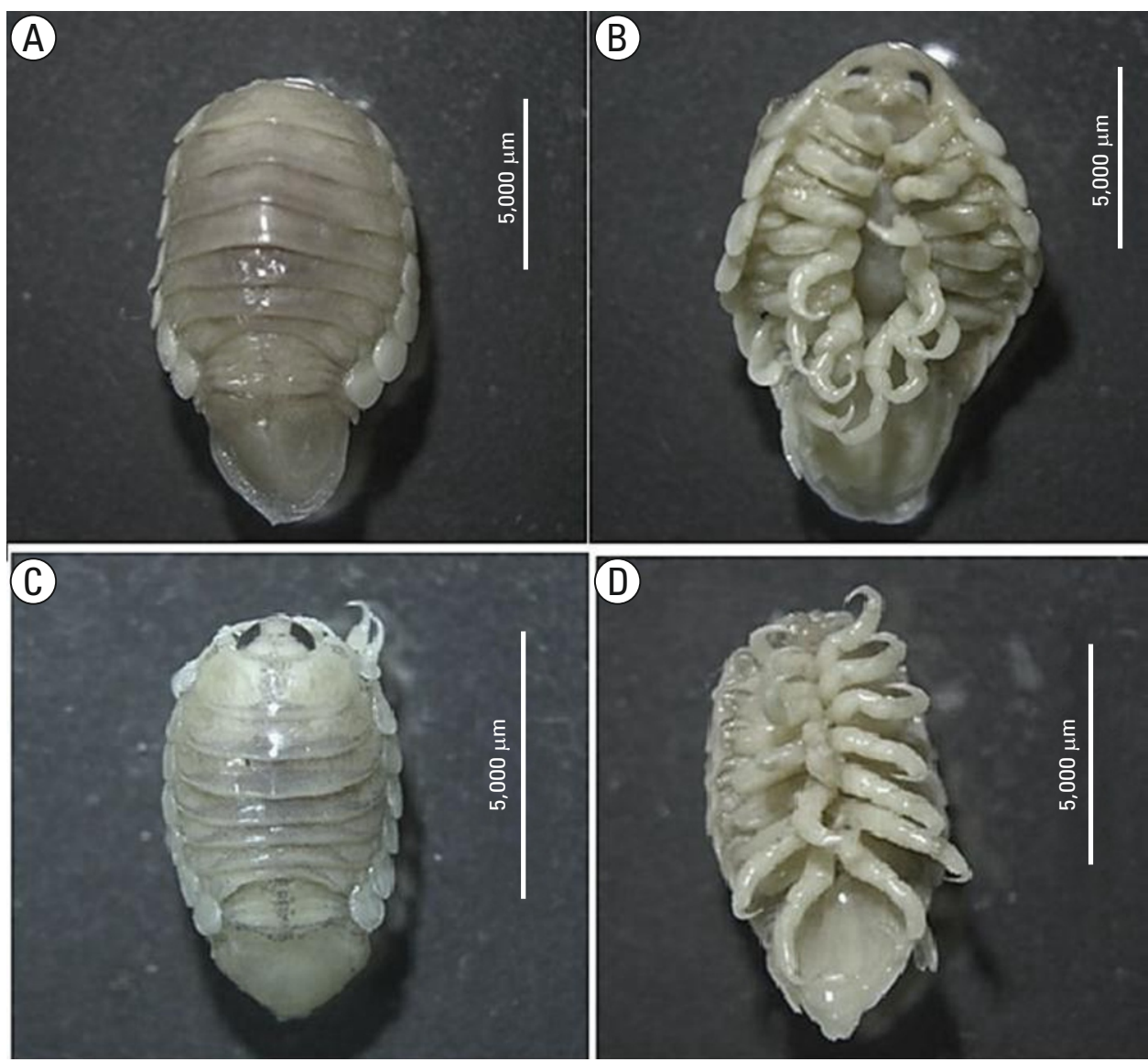

Figura 2. Anphira branchialis. A. Vista dorsal da fêmea. B. Vista ventral da fêmea. C. Vista dorsal do macho, D. Vista ventral do macho. Escalas das barras $5 \mathrm{~mm}$. 
Comentários: no presente trabalho a morfologia dos espécimes analisados de $A$. branchialis estão de acordo com os descritos em Thatcher (1993). Quanto à morfometria, no presente trabalho a média do comprimento e largura dos indivíduos analisados foi menor que as reportadas por Thatcher (1993) e Morais (2012). Os valores mínimos e máximos se encaixaram dentro dos valores registrados por aqueles autores.

Anphira branchialis é distinguida de outros gêneros e espécies de Cymothoidae pela presença de grandes placas coxais sobrepostas em todos os sete pereonitos que normalmente, não ocorrem no primeiro pereonito para membros desta família (Thatcher, 1993) (Figura 3)
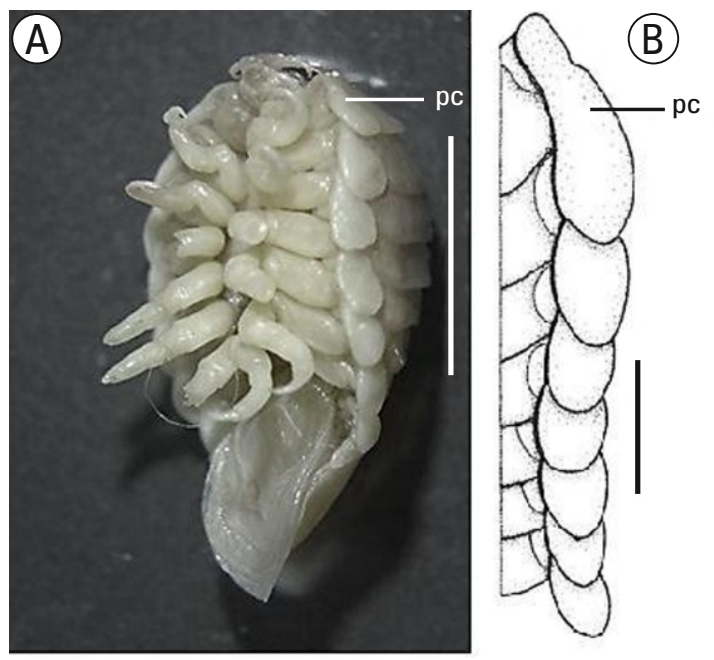

Figura 3. Anphira branchialis. A. Vista lateral mostrando as placas coxais nos sete perionitos, Escala da barra: $5 \mathrm{~mm}$. B. Esquema das placas coxais. Escala da barra: $2 \mathrm{~mm}$.

Vanamea symmetrica (Van Name, 1925) (Figura 4). Descrição baseada em um macho encontrado na cavidade branquial: Corpo longo, simétrico, comprimento ultrapassa duas vezes a largura, cor amarelado. Comprimento do corpo: $10.34 \mathrm{~mm}$, largura: 4.51. Cefalón não imerso no pereonito1; pereonito 4 mais alto e mais largo que os demais; pereonito 1 mais comprido que os demais, com projeções anteriores. Peças bucais: mandíbula pediforme, serrilhada medianamente, processo de corte longo, com comprimento correspondente aos dois primeiros artículos do palpo; maxilípede com lóbulos laterais provido de cerdas pectinadas. Pereópodos de todas as pernas, e no carpo da perna 7; dátilo da perna 7 menor que os demais. Pleón em forma de língua; pleonitos bem evidentes; pleotelso mais largo que longo, inflado, quilhado dorsal e

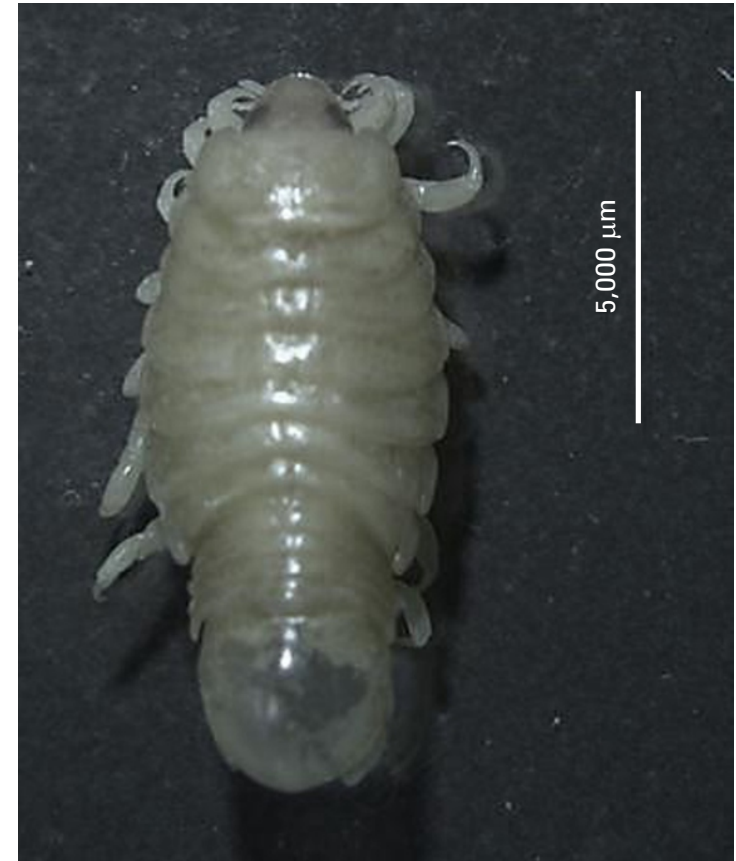

Figura 4. Vista dorsal do macho de Vanamea symetrica. Escala da barra: $5 \mathrm{~mm}$.

medianamente. Urópodo menor ou igual ao comprimento do pleotelso. Comprimento do pleotelso: 2.48 e largura: 2.91. Endopodito menor que o exopodito e com cerdas.

Comentários: $V$. symmetrica foi descrita no gênero Livoneca por Van Name (1925). Devido a razões morfológicas, Bruce (1990) retirou a espécie Livone casymmetrica do gênero. A espécie foi proposta em um novo gênero (Vanamea) por Thatcher (1993).

Esta espécie é diferenciada dos outros gêneros de Isopoda parasitas de peixes de água doce, principalmente pela forma do corpo, os peréopodos alongados e o pleotelso inflado. No presente trabalho a morfologia dos espécimes analisados de A. branchialis estão de acordo com os descritos em Thatcher (1993). Quanto à morfometria, o comprimento e largura dos indivíduos analisados foram menores que as reportadas por Thatcher (1993).

\section{DISCUSSÃO}

Em peixes da família Serrasalmidae o Isopoda Amphira branchialis foi registrado em $P$. nattereri (Thatcher, 1993; Carvalho et al., 2004; Vital et al., 2011; Morais, 2012, Tavares-Dias et al., 2015), em S. spilopleura e em Serrasalmus sp. (Thatcher, 1993, Tavares-Dias et al., 2015). Vanamea symetrica foi 
registrado em $S$. elongatus, $S$. rhombeus, $S$. spilopleura e em Serrasalmus sp. (Thatcher, 1993). No presente estudo as espécies $A$. branchialis e $V$. symetrica foram registradas pela primeira vez parasitando as brânquias de $S$. altispinis aumentando assim o número de hospedeiros conhecidos para estas espécies de parasitas.

Nos isópodos, os sexos são separados e a fertilização é interna. Machos e fêmeas da família Cymothoidae são encontrados frequentemente, juntos no mesmo individuo. Acredita-se que todos os cymothoideos sejam hermafroditas protândricos. Dessa forma, o cymothoideo uma vez que tenha se localizado no hospedeiro (peixe) modifica sua morfologia e sexualidade de macho para fêmea. $\mathrm{O}$ segundo cymothoideo no momento da fixação permanece com a morfologia do macho quando encontra uma fêmea no hospedeiro (Machado et al., 1996; Pavanelli, 1999). No presente estudo em oito hospedeiros parasitados por Amphira branchialis, sete estavam parasitados por um macho e uma fêmea no mesmo hospedeiro, e um peixe estava parasitado por dois indivíduos machos, confirmando assim a protandria neste grupo de crustáceos.

A patogenicidade do isópodo varia de acordo com o seu comportamento alimentar, com seu tamanho e com sua estratégia de ataque no hospedeiro. Quatro áreas de ataque e de fixações podem ser encontradas, como por exemplo, a pele ou as nadadeiras, a cavidade branquial, a boca e a língua e as cavidades ou bolsas que eles produzem dentro da cavidade do corpo. Cada um desses locais sofre modificações com a presença dos isópodos (Brusca \& Wilson, 1991; Rhode, 2005).

Os isópodos que habitam a câmara branquial localizam-se sobre os filamentos branquiais, onde se alimentam, sendo encontrados em seus intestinos, células sanguíneas do hospedeiro. Além da perda de sangue que o isópodo ocasiona no hospedeiro, ocorre também à redução do número de filamentos branquiais, com a diminuição da eficiência respiratória do peixe (Machado et al., 1996; Pavanelli, 1999).

No presente estudo os isópodos foram registrados parasitando as brânquias de $S$. altispinis, os quais apresentaram perda e redução dos filamentos brânquias, o que pode ser atribuído à infestação por estes parasitas.

Para os peixes, o fator de condição relativo $(\mathrm{Kn})$ é uma medida ou indicador do bem-estar que fornece informações relevantes sobre as condições nutricionais recentes, gastos de reservas, infecções parasitárias e outros (Vazzoler, 1996). Tem sido relatado que o peixe parasitado por isópodos terá seu metabolismo, crescimento e reprodução prejudicados (Rhode, 2005). No presente trabalho, de acordo com o fator de condição relativo, os peixes parasitados por isópodes apresentaram um fator de condição médio considerado como normal, descartando alguma influência negativa destes parasitas relacionada com o crescimento, peso ou fator de condição.

Os dados obtidos no presente trabalho aumentam o conhecimento das espécies parasitas de $S$. altispinis, sendo reportadas duas novas espécies que a parasitam e duas novas ocorrências de $A$. branquialis e $V$. symetrica em um novo hospedeiro proveniente de lagos de várzea da Amazônia brasileira.

\section{REFERÊNCIAS BIBLIOGRÁFICAS}

Amato, J.F.R. 1991. Protocolos para laboratório coleta e processamento de parasitas do pescado. Imprensa Universitária, Universidade Federal do Rio de Janeiro, Rio de Janeiro, Brasil. 81 p.

Bush, A.O.; Lafferty, K.D.; Lotz, J.M.; Shostak, A.W. 1997. Parasitology meets ecology on its own terms: Margolis et al. Revisited. Jornaul of Parasitology, 83(4): 575 - 583.

Brusca, R.C.; Wilson, G.D.F. 1991. A philogenetic analysis of the Isopoda with some classificatory recommendations. Memoirs of the Queensland Museum, 31: 143-204.

Carvalho, L. N.; Arruda, R.; Del-Claro, K. (2004). Host-parasite interactions between the piranha Pygocentrus nattereri (Characiformes: Characidae) and isopods and branchiurans (Crustacea) in the rio Araguaia basin, Brazil. Neotropical Ichthyology,2(2): 93-98.

Claro Jr., L.H. 2003. A influência da floresta alagada na estrutura trófica de comunidades de peixes em lagos de várzea da Amazônia Central. Dissertação de Mesrado, INPA-UFAM. Manaus, Amazonas. 61pp.

Eiras, J.C.; Takemoto, R.M.; Pavanelli, G.C. 2000. Métodos de estudo e técnicas laboratoriais em parasitologia de peixes. Editora da Universidade Estadual de Maringá, PR, Brasil. 173pp.

Eiras, J.C.; Takemoto, R.M.; Pavanelli, G.C.; Adriano, E.A. 2010. Diversidade dos parasitas de peixes de água doce do Brasil. Maringá: Clichetec, 2380-2389.

Froese, R.; Pauly, D. 2014. FishBase. World Wide Web eletronic publication. www.fishbase.org, version 2014.

Le Cren, E.D. 1951. The length-weight relationship and seasonal cycle in gonadal weight and condition in the perch (Percafluviatilis). Journal 
of Animal Ecology, New York, 20: 201-219.

Luque, J.L.; Poulin, R. 2007. Metazoan parasite species richness in Neotropical fishes: hotspots and the geography of biodiversity. Parasitology, $134(6): 865-878$.

Machado, M. H.; Pavanelli, G.C.; Takemoto, R. M. 1996. Introdução ao estudo dos parasitas de peixes. Ed. Universidade Estadual de Maringá, PR. 69pp.

Möler, H.; Anders, K. 1986. Diseases and parasites of marine fish. Verlag Heino Möller, Kiel. 365p.

Morais, A.M. 2012. Biodiversidade de parasitos da piranha vermelha Pygocentrus nattereri (Kner, 1858) (Characiformes; Serrasalmidae) e sua avaliação como bioindicadores na Amazônia Central. Tese de Doutorado, Instituto Nacional de Pesquisas da Amazônia, Manaus, Amazonas. $234 \mathrm{p}$.

Morey, G.M.; Malta, J.C.O. 2016. As espécies de copepoda (Crustacea: Ergasilidae) parasitas dos filamentos branquiais e narinas de Serrasalmus altispinis (Merckx, Jegu e Santos, 2000) (Characiformes - Serrasalmidae) em lagos de várzea do Brasil. Folia Amazonica, 25 (1): 5560.

Pavanelli, G.C.; Eiras, J.C.; Takemoto, R.M. 1999. Doenças de Peixes. Profilaxia, Diagnóstico e Tratamento. $2^{\mathrm{a}}$ Ed. Universidade Estadual de Maringá, Maringá, 305p.

Ramdane, Z.; Bensouila, M.A.; Trilles, J.P. 2007. The Cymothoidae (Crustacea, Isopoda), parasites on marine fishes, from Algerian fauna. Belgian Journal of Zoology, 137(1): 67-74.
Rohde, K.(ED). 2005. Marine Parasitology. Austrália: CSIRO publishing, Collingwood. Victoria CABI. 592p.

Tavares-Dias, M.; Dias-Júnior, M.B.F.; Florentino, A.C.; Silva, L.M.A.; Cunha, A.C.D. 2015. Distribution pattern of crustacean ectoparasites of freshwater fish from Brazil. Revista Brasileira de Parasitologia Veterinária, 24(2): 136-147.

Thatcher, V.E. 1993. Vanamea Gen, Nov. For Livoneca symmetrica Van Name, 1925, (Crustacea, Isopoda, Cymothoidae) and a redescription of the species based on specimens from Brazilian piranhas. Acta Amazonica, 23(23): 287-296.

Vazzoler, A.E.A.M.; Menezes, N.A. 1992. Síntese dos conhecimentos sobre o comportamento reprodutivo dos Characiformes da América do Sul (Teleostei, Osthariophysi). Revista Brasileira de biologia, Rio de Janeiro, 52(4): 627-640.

Vital, J.F.; Varella, A.M.B.; Porto, D.B.; Malta, J.C.O. 2011. Sazonalidade da fauna de metazoários de Pygocentrus nattereri (Kner, 1858) no lago Piranha (Amazonas, Brasil) e a avaliação de seu potencial como indicadora da saúde do ambiente. Biota Neotropical, 11(1): 199-204.

Recibido: 23 de Junio del 2016

Aceptado para publicación: 2 de Agosto del 2016 
